\title{
Phase Diagram of the Dirac Spectrum at Nonzero Chemical Potential
}

\author{
J.C. Osborn, ${ }^{1,2}$ K. Splittorff, ${ }^{3}$ and J.J.M. Verbaarschot ${ }^{4}$ \\ ${ }^{1}$ Argonne Leadership Computing Facility, 9700 S. Cass Avenue, Argonne, IL 60439, USA \\ ${ }^{2}$ Center for Computational Science, Boston University, Boston, MA 02215, USA \\ ${ }^{3}$ The Niels Bohr Institute, Blegdamsvej 17, DK-2100, Copenhagen Ø, Denmark \\ ${ }^{4}$ Department of Physics and Astronomy, SUNY, Stony Brook, New York 11794, USA
}

(Dated: September 22, 2018)

\begin{abstract}
The Dirac spectrum of QCD with dynamical fermions at nonzero chemical potential is characterized by three regions, a region with a constant eigenvalue density, a region where the eigenvalue density shows oscillations that grow exponentially with the volume and the remainder of the complex plane where the eigenvalue density is zero. In this paper we derive the phase diagram of the Dirac spectrum from a chiral Lagrangian. We show that the constant eigenvalue density corresponds to a pion condensed phase while the strongly oscillating region is given by a kaon condensed phase. The normal phase with nonzero chiral condensate but vanishing Bose condensates coincides with the region of the complex plane where there are no eigenvalues.
\end{abstract}




\section{INTRODUCTION}

Because of the Banks-Casher relation [1] between the chiral condensate and the spectral density of the QCD Dirac operator, lattice QCD studies have had a long lasting interest in the Dirac spectrum. The eigenvalue correlations have been well understood for an (anti-)Hermitian Dirac operator. In the chirally broken phase, the eigenvalue density around zero is nonzero in the thermodynamic limit resulting in a discontinuity of the chiral condensate. In the chirally restored phase, a gap develops around zero so that the condensate remains a smooth function of the mass in the thermodynamic limit and therefore vanishes in the massless limit.

At nonzero quark chemical potential, $\mu$, the Dirac spectrum also received considerable attention in the lattice QCD literature [2, 3, 4, 5, 6, 7, 8, 9, 10]. In quenched lattice simulations it was found that the Dirac eigenvalues are distributed more or less homogeneously inside a strip that is symmetric about the imaginary axis. In particular, this implies that the quenched chiral condensate vanishes in the massless limit for arbitrarily small chemical potential [2]. On the other hand, because at very low temperatures the free energy of QCD with dynamical quarks does not depend on the quark chemical potential below a third of the nucleon mass, chiral symmetry must remain broken at small nonzero chemical potential if it is broken at zero chemical potential. Naively, the fermion determinant will make matters worse (an even smoother behavior of the condensate at $m=0$ ) because eigenvalues will be repelled from the position of the mass [2], but as will be discussed below, it is precisely the phase of the fermion determinant that will lead to a nonzero chiral condensate.

A partial resolution of this paradox appeared [3] soon after the publication of [2]. Based on a onedimensional $U(1)$ lattice model it was concluded that phase fluctuations of the quark determinant are essential to obtain a nonzero chiral condensate. In the same paper, it was found that the width of the strip of eigenvalues is equal to the quark mass when $\mu=m_{\pi} / 2$. This has been verified by lattice simulations [6, 7]. Recently, this result was derived from a chiral Lagrangian for the Dirac

spectrum of quenched QCD [11]. However, the paradox of how a $\mu$-dependent spectral density can lead to both a $\mu$-independent partition function and a discontinuity of the chiral condensate remained unsolved and was later coined [12] as the "Silver Blaze Problem."

The resolution of the "Silver Blaze Problem" became possible because of two further developments. First, the idea to explain the failure of the quenched approximation at $\mu \neq 0$ in a random matrix model with the symmetries of QCD at $\mu=0$ [13]. Second, the introduction of a mathematically simpler version of this model that made it possible to derive exact analytical expressions 
for the spectral density of the QCD Dirac operator with dynamical quarks [14, 15]. Using these results it was shown in [16, 17] that a strongly oscillating contribution to the spectral density is responsible for the discontinuity in the chiral condensate.

The random matrix results are valid for QCD in the microscopic limit [18, 19] where the combinations

$$
\hat{m} \equiv m \Sigma V \quad \text { and } \quad \hat{\mu}^{2} \equiv \mu^{2} F^{2} V
$$

are kept fixed in the thermodynamic limit (Here, $m$ denotes any of the quark masses or the mass scale for which the Dirac spectrum is calculated, $\mu$ is the chemical potential, $\Sigma$ is the magnitude of the chiral condensate and $F$ is the pion decay constant). Since in this limit, the contribution of the zero momentum modes factorizes from the partition function [20], it is also equivalent to the $\epsilon$ regime of chiral perturbation theory [21]. Random matrix predictions for the quenched spectral density 22, 23] have been compared successfully to lattice gauge simulations for the microscopic domain [24, 25, 26].

In this paper we show that the microscopic results for the unquenched Dirac spectrum are characterized by three different regions or "phases": a phase where there are no eigenvalues, a phase where the eigenvalue density is equal to the quenched result, and a phase where the Dirac spectrum is strongly oscillating. The aim of the present paper is to derive the phases of the Dirac spectrum from a mean field analysis of the chiral Lagrangian for the Dirac spectrum of QCD at $\mu \neq 0$.

In the mean field limit one evaluates the extrema of the static part of the chiral Lagrangian so that the leading order contribution in the $p$-expansion of chiral perturbation theory is exactly given by the large $\hat{m}$ and large $\hat{\mu}$ limit of the microscopic limit of QCD. The classification of the spectral density of the Dirac operator is therefore valid beyond the microscopic regime up to energies that are well below the QCD scale. Alternatively, the locus of these phases in the complex eigenvalue plane can be obtained [16] from the asymptotic properties of the complex Laguerre polynomials used to solve the corresponding random matrix model.

In section $\amalg$ we review the calculation of the phase diagram of the quenched Dirac spectrum. The phase diagram of the Dirac spectrum for QCD with one dynamical flavor is discussed in section III. and the dependence of the free energy on the number of replicas is discussed in section IV] Concluding remarks are made in section IV] A brief discussion of some of the results of this paper appeared in the proceedings [27]. 


\section{PHASE DIAGRAM FOR THE QUENCHED DIRAC SPECTRUM}

In this section we review the mean field analysis of the phases of the quenched Dirac spectrum originally given in [11] using the supersymmetric method. In this paper we evaluate the quenched spectral density by means of the replica trick given by [13, 28]

$$
\begin{aligned}
\rho^{N_{f}=0}\left(z, z^{*} ; \mu\right) & =\lim _{n \rightarrow 0} \frac{1}{V} \frac{1}{n} \partial_{z} \partial_{z^{*}} \log Z^{n}\left(z, z^{*} ; \mu\right), \\
Z^{n}\left(z, z^{*} ; \mu\right) & =\left\langle\operatorname{det}^{n}\left(D+\gamma_{0} \mu+z\right) \operatorname{det}^{n}\left(D-\gamma_{0} \mu+z^{*}\right)\right\rangle,
\end{aligned}
$$

where $\langle\ldots\rangle$ denotes the quenched ensemble average. In the mean field limit the result for $\rho^{N_{f}=0}\left(z, z^{*} ; \mu\right)$ before taking the replica limit, $n \rightarrow 0$, does not depend on $n$ and the limit $n \rightarrow 0$ can be taken trivially. Therefore it is sufficient to analyze the partition function for $n=1$ which, as is illustrated by the above rewriting of the partition function, is equivalent to QCD at nonzero isospin chemical potential with complex quark masses. The replica argument can be made rigorous in the microscopic limit [29, 30] through the Toda lattice equation which links the generating functionals, $Z^{n}$ with different replica index $n$. Using the Toda lattice equation one can derive an exact identity [15, 22] for the quenched microscopic spectral density in the sector with topological charge $\nu$

$$
\rho^{N_{f}=0}\left(\hat{z}, \hat{z}^{*} ; \hat{\mu}\right)=\frac{1}{2} \hat{z} \hat{z}^{*} Z^{n=1}\left(\hat{z}, \hat{z}^{*} ; \hat{\mu}\right) Z^{n=-1}\left(\hat{z}, \hat{z}^{*} ; \hat{\mu}\right) .
$$

Since the microscopic limit of the partition function with a pair of conjugate bosonic quarks, $Z^{n=-1}\left(z, z^{*} ; \mu\right)$, given by [15, 22]

$$
Z^{n=-1}\left(\hat{z}, \hat{z}^{*} ; \hat{\mu}\right)=|\hat{z}|^{2 \nu} \frac{1}{\hat{\mu}^{2}} \exp \left(-\frac{\left(\hat{z}^{2}+\hat{z}^{* 2}\right)}{8 \hat{\mu}^{2}}\right) K_{\nu}\left(\frac{|\hat{z}|^{2}}{4 \hat{\mu}^{2}}\right)
$$

is a smooth function of $z$, the phase structure of the spectral density is given by $Z^{n=1}\left(z, z^{*} ; \mu\right)$ (in agreement with the conclusion from the mean field replica trick).

We will examine the partition function $Z^{n=1}\left(z, z^{*} ; \mu\right)$ to leading order in chiral perturbation theory. The corresponding chiral Lagrangian is given by [11, 31]

$$
\mathcal{L}_{\text {eff }}=\frac{1}{4} F^{2} \operatorname{Tr} \nabla_{\nu} U \nabla_{\nu} U^{\dagger}-\frac{1}{2} \Sigma \operatorname{Tr} M\left(U+U^{\dagger}\right),
$$

with $U \in U(2)$ and covariant derivative defined by

$$
\nabla_{\nu} U=\partial_{\nu} U-i\left[B_{\nu}, U\right]
$$




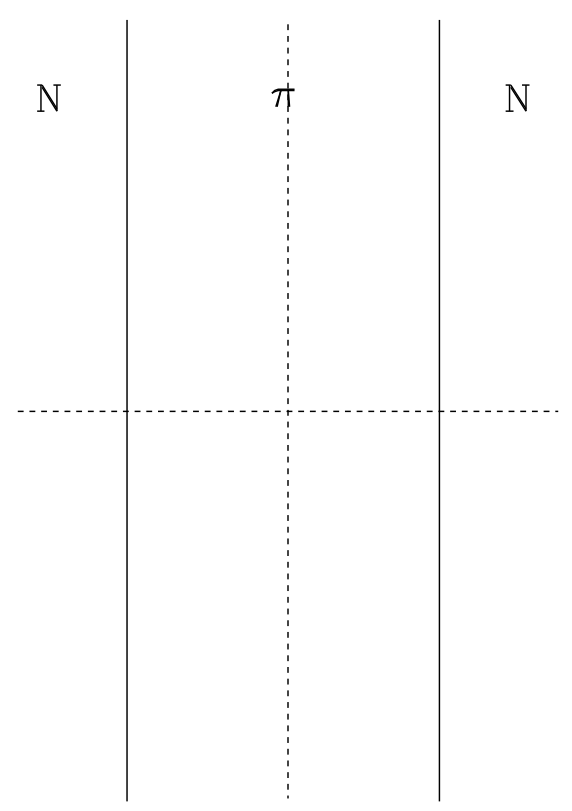

FIG. 1: Phase diagram of the quenched Dirac spectrum in the complex $z$ plane. The support of the quenched spectrum is between the two solid vertical black lines. The labels $\mathrm{N}$ and $\pi$ refer to the normal and pion condensed phase, respectively.

with

$$
B_{\nu}=\delta_{\nu, 0}\left(\begin{array}{cc}
\mu & 0 \\
0 & -\mu
\end{array}\right) \quad \text { and } \quad M=\left(\begin{array}{cc}
z & 0 \\
0 & z^{*}
\end{array}\right)
$$

We can neglect higher order terms in $\mu$ as long as $\mu \ll F$. In the mean field limit, the phase structure of the partition function is determined by the static part of the Lagrangian given by

$$
\mathcal{L}_{\text {stat }}=\frac{1}{4} F^{2} \operatorname{Tr}[U, B]\left[U^{\dagger}, B\right]-\frac{1}{2} \Sigma \operatorname{Tr} M\left(U+U^{\dagger}\right) .
$$

Notice that $V \mathcal{L}_{\text {stat }}$ only depends on the microscopic variables (11). In order for the mean field limit to be valid for the calculation of bulk quantities, we also require that $m,|z| \ll F^{4} / \Sigma$.

The ground state of this Lagrangian is easily found by making the ansatz

$$
U=\left(\begin{array}{cc}
\cos \alpha & \sin \alpha \\
-\sin \alpha & \cos \alpha
\end{array}\right)
$$

This results in the effective Lagrangian

$$
\mathcal{L}_{\text {stat }}=-2 \mu^{2} F^{2} \sin ^{2} \alpha-\Sigma\left(z+z^{*}\right) \cos \alpha .
$$


The minima of this Lagrangian are given by

$$
\sin \alpha=0 \quad \text { and } \quad \cos \alpha=\frac{\Sigma\left(z+z^{*}\right)}{4 \mu^{2} F^{2}}
$$

resulting in the free energies

$$
\mathcal{L}_{\text {stat }}^{\mathrm{N}}=-\Sigma\left(z+z^{*}\right) \quad \text { and } \quad \mathcal{L}_{\text {stat }}^{\pi}=-2 \mu^{2} F^{2}-\frac{\Sigma^{2}\left(z+z^{*}\right)^{2}}{8 \mu^{2} F^{2}},
$$

respectively. The boundary between the two phases is given the condition

$$
\Sigma\left(z+z^{*}\right)=4 \mu^{2} F^{2} .
$$

This condition can easily be understood physically. Since the mass of the charged Goldstone bosons is given by

$$
m_{\pi}^{2}=\frac{\left(z+z^{*}\right) \Sigma}{F^{2}}
$$

pion condensation occurs when $\mu^{2}>\left(m_{\pi} / 2\right)^{2}=\left(z+z^{*}\right) \Sigma /\left(4 F^{2}\right)$.

A schematic phase diagram of the quenched Dirac spectrum is shown in Fig. 1). The normal phase $(\mathrm{N})$, where $\sin \alpha=0$, and the pion condensed phase, where $\cos \alpha=\Sigma\left(z+z^{*}\right) /\left(4 \mu^{2} F^{2}\right)$, occur for $\left|\left(z+z^{*}\right) \Sigma /\left(4 F^{2}\right)\right|>\mu^{2}$ and $\left|\left(z+z^{*}\right) \Sigma /\left(4 F^{2}\right)\right|<\mu^{2}$, respectively. Inside the pion condensation region the eigenvalue density (per unit area) is constant

$$
\rho_{\pi}^{N_{f}=0}\left(z, z^{*} ; \mu\right)=-\frac{1}{\pi} \partial_{z} \partial_{z^{*}} \mathcal{L}_{\text {stat }}^{\pi}=\frac{\Sigma^{2}}{4 \mu^{2} F^{2}},
$$

while outside this region the eigenvalue density is zero $\rho\left(|x|>2 \mu^{2} F^{2} / \Sigma\right)=-\partial_{z} \partial_{z^{*}} \mathcal{L}_{\text {stat }}^{\mathrm{N}}=0$.

\section{PHASE DIAGRAM OF THE DIRAC SPECTRUM FOR QCD WITH DYNAMICAL QUARKS}

In this section we discuss the phase diagram of the Dirac spectrum of QCD with dynamical quarks at nonzero chemical potential. We will do this by relating our results to strangeness condensation and pion condensation at nonzero quark chemical potentials, a connection that was mentioned in [16] and discussed briefly in the proceedings [27].

The discontinuity of the chiral condensate arises due to the contribution from the strongly oscillating terms in the spectral density [16, 17]. These terms become exponentially large with increasing volume, but are exponentially suppressed elsewhere [14, 15]. Here we will show that this region can be identified from the mean field limit of the chiral Lagrangian and it therefore shows that our picture of the Dirac spectrum is valid beyond the microscopic domain. 
As in the quenched case we use the replica trick to express the unquenched eigenvalue density

$$
\rho^{N_{f}}\left(z, z^{*}, m ; \mu\right)=\lim _{n \rightarrow 0} \frac{1}{V} \frac{1}{n} \partial_{z} \partial_{z^{*}} \log Z^{n, N_{f}}\left(z, z^{*}, m ; \mu\right),
$$

in terms of generating functionals for the eigenvalue density

$$
Z^{n, N_{f}}\left(z, z^{*}, m ; \mu\right)=\left\langle\operatorname{det}^{n}\left(D+\gamma_{0} \mu+z\right) \operatorname{det}^{n}\left(D-\gamma_{0} \mu+z^{*}\right) \operatorname{det}^{N_{f}}\left(D+\gamma_{0} \mu+m\right)\right\rangle .
$$

Below we will see that in the mean field limit of the chiral Lagrangian, the free energy does not always scale with $n$ as in the case of the phase quenched partition function. Therefore, the naive replica trick does not necessarily work in this case. However, it turns out (see section IV) that the boundary of the the oscillating region as well as the boundary of the region with a constant eigenvalues density do not depend on $n$. Therefore, these boundaries can be obtained from a calculation for $n=1$ which will be discussed in the remainder of this section. In the microscopic limit this also follows from an exact identity (that can be derived from the Toda lattice equation) between the spectral density and the ratio of the the following partition functions (recall that the microscopic variables defined in (1) are denoted by a hat) [15]

$$
\rho^{N_{f}}\left(\hat{z}, \hat{z}^{*}, \hat{m} ; \hat{\mu}\right)=\frac{\hat{z} \hat{z}^{*}}{2}\left(\hat{m}^{2}-\hat{z}^{2}\right)^{N_{f}} Z^{n=-1}\left(\hat{z}, \hat{z}^{*} ; \hat{\mu}\right) \frac{Z^{N_{f}, n=1}\left(\hat{m}, \hat{z}, \hat{z}^{*} ; \hat{\mu}\right)}{Z^{N_{f}}(\hat{m} ; \hat{\mu})} .
$$

Here, the label $N_{f}$ denotes the number of fermionic quark flavors with mass $m$ and the value of the label $n$ stands for the number of quark pairs with mass $z$ and $z^{*}$ (a negative value of $n$ should be interpreted as $n$ pairs of bosonic quarks). Since both the microscopic limit of $Z^{n=-1}\left(z, z^{*} ; \mu\right)$ given in (4) and the $N_{f}$ flavor partition $Z^{N_{f}}(\hat{m} ; \hat{\mu})=Z^{N_{f}}(\hat{m})$ do not show a phase transition in the chemical potential-mass plane, the phase structure of the spectral density is determined by $Z^{N_{f}, n=1}\left(\hat{m}, \hat{z}, \hat{z}^{*} ; \hat{\mu}\right)$. Note that the oscillating nature must come from $Z^{N_{f}, n=1}\left(\hat{m}, \hat{z}, \hat{z}^{*} ; \hat{\mu}\right)$ since both $Z^{n=-1}\left(\hat{z}, \hat{z}^{*} ; \hat{\mu}\right)$ and $Z^{N_{f}}(\hat{m})$ are real and positive. The phase structure can be analyzed in the thermodynamic limit which is smoothly connected to the strong non-Hermiticity limit $(\hat{\mu} \gg 1)$ of the microscopic domain. In the large $\hat{\mu}$ limit with $\hat{m} \sim \hat{\mu}^{2}$ and $|\hat{z}| \sim \hat{\mu}^{2}$ we have that

$$
\rho^{N_{f}}\left(\hat{z}, \hat{z}^{*}, \hat{m} ; \hat{\mu}\right) e^{2 \hat{\mu}^{2}+\hat{x}^{2} /\left(2 \hat{\mu}^{2}\right)+N_{f} \hat{m}} \sim Z^{N_{f}, n=1}\left(\hat{m}, \hat{z}, \hat{z}^{*} ; \mu\right) .
$$

Below we will evaluate the partition function $Z^{N_{f}, n=1}\left(m, z, z^{*} ; \mu\right)$ in the mean field limit. The phase structure does not depend on the number of flavors, and we will only study the case $N_{f}=1$. Alternatively, one could analyze the strong non-Hermiticity limit of the spectral density [16] where the phases are determined by a competition between the quenched and unquenched contribution, and whether a saddle point of the expression hits the boundary on the integration interval. 


\section{A. Low Energy Limit of QCD Partition Function}

The QCD generating functional with $n=1$ for the $N_{f}=1$ spectral density is given by the partition function

$$
\begin{aligned}
Z^{N_{f}=1, n=1}\left(m, z, z^{*} ; \mu\right) & =\left\langle\operatorname{det}\left(D+\mu \gamma_{0}+m\right) \operatorname{det}\left(D+\mu \gamma_{0}+z\right) \operatorname{det}\left(D^{\dagger}+\mu \gamma_{0}+z^{*}\right)\right\rangle \\
& =\left\langle\operatorname{det}\left(D+\mu \gamma_{0}+m\right) \operatorname{det}\left(D+\mu \gamma_{0}+z\right) \operatorname{det}\left(D-\mu \gamma_{0}+z^{*}\right)\right\rangle
\end{aligned}
$$

We can interpret the flavor with mass $m$ as the strange flavor and $z$ and $z^{*}$ as the up and down flavors. The isospin and strangeness chemical potential are then given by

$$
\mu_{I}=2 \mu, \quad \mu_{S}=\mu
$$

The quark chemical potential $\mu$ of the generating functional for the unquenched eigenvalue density thus couples to the charges carried by the pions and the kaons. That is why the chiral Lagrangian can give us nontrivial information about the Dirac spectrum even though the Goldstone bosons have zero baryon charge. The masses of the the Goldstone bosons are equal to

$$
m_{K}^{2}=\operatorname{Re} \Sigma\left(m+z^{*}\right) / F^{2}, \quad m_{\pi}^{2}=\Sigma\left(z+z^{*}\right) / F^{2} .
$$

For $m \rightarrow \infty$ the partition function (20) reduces to the quenched case discussed in the previous section. In this case it was found [11, 13] that the phase with the pion condensate corresponds to the region where the eigenvalue density is nonzero whereas the region with vanishing eigenvalue density is in the normal phase (see eq. (15)). As we lower the strange quark mass $m$, at some point the mass of the kaon is less than that of the pion, which leads to the formation of a kaon condensate [32]. Given $m$, we will see below that the values of $z$ and $z^{*}$ for which the kaon condensate is nonzero will correspond to a region in the complex eigenvalue plane where the unquenched eigenvalue density is oscillating with and amplitude that grows exponentially with the volume.

The low-energy limit of the partition function (20) for real $z$ was analyzed in [32]. Here we extend the arguments to the complex plane. The chiral Lagrangian for the Goldstone fields is given by the same expression as in the phase quenched case,

$$
\mathcal{L}_{\text {eff }}=\frac{1}{4} F^{2} \operatorname{Tr} \nabla_{\nu} U \nabla_{\nu} U^{\dagger}-\frac{1}{2} \Sigma \operatorname{Tr} M\left(U+U^{\dagger}\right),
$$

with covariant derivative defined by

$$
\nabla_{\nu} U=\partial_{\nu} U-i\left[B_{\nu}, U\right]
$$


but in this case

$$
\begin{aligned}
B_{\nu} & =\delta_{\nu, 0} \operatorname{diag}\left(\frac{1}{3} \mu_{B}+\frac{1}{2} \mu_{I}, \frac{1}{3} \mu_{B}-\frac{1}{2} \mu_{I}, \frac{1}{3} \mu_{B}+\mu_{S}\right), \\
\mathcal{M} & =\operatorname{diag}\left(z, z^{*}, m\right),
\end{aligned}
$$

and $U \in S U(3)$. The phases are determined by the vacuum states that minimize the free energy. In the mean field limit it is sufficient to consider the zero momentum modes or constant fields, and we only have to minimize the static part of the effective Lagrangian given by

$$
\mathcal{L}_{\text {stat }}=\frac{1}{4} F^{2} \operatorname{Tr}[U, B]\left[U^{\dagger}, B\right]-\frac{1}{2} \Sigma \operatorname{Tr} M\left(U+U^{\dagger}\right) .
$$

\section{B. Vacuum Configurations}

The ground states of (27) can be parameterized by the ansatz

$$
\bar{U}=R_{y}(-\beta) R_{z}(\alpha) R_{y}(\beta)
$$

where $R_{x}(\beta)$ is a rotation by $\beta$ about the $x$-axis, etc.. Because of (21) we have $B_{0}=\mu \operatorname{diag}(1,-1,1)$ and the static part of the Lagrangian becomes

$$
\mathcal{L}_{\text {stat }}=-2 \mu^{2} F^{2}\left(1-\cos ^{2} \alpha\right)-\Sigma\left[(z-m) \cos ^{2} \beta(\cos \alpha-1)+z+z^{*} \cos \alpha+m \cos \alpha\right] .
$$

The ground state is given by the minimum of the real and imaginary parts of the Lagrangian. For definiteness we take $m>0$ and $x \equiv \operatorname{Re}(z)>0$. For $\alpha=0$ the value of $\beta$ is not fixed. This phase, where $\bar{U}=1$, is the normal phase with free energy given by

$$
\mathcal{L}_{\text {stat }}^{N}=-\Sigma\left(m+z+z^{*}\right)
$$

For $\alpha \neq 0$, the minimum of $\mathcal{L}_{\text {stat }}$ is either at $\beta=0$ or at $\beta=\pi / 2$. For $\beta=0$ we find

$$
\cos \alpha=\frac{\Sigma\left(z+z^{*}\right)}{4 \mu^{2} F^{2}} .
$$

This is the pion condensed phase with free energy given by

$$
\mathcal{L}_{\text {stat }}^{\pi}=-2 \mu^{2} F^{2}\left(1-\cos ^{2} \alpha\right)-\Sigma\left(z+z^{*}\right) \cos \alpha-\Sigma m=-\Sigma m-2 \mu^{2} F^{2}-\frac{\Sigma^{2}\left(z+z^{*}\right)^{2}}{8 \mu^{2} F^{2}} .
$$

This expression also enters in the relation between the spectral density and the partition function in the strong non-Hermiticity limit (see Eq. (19)), and can be concisely written as

$$
\rho_{\nu}^{N_{f}=1}\left(\hat{z}, \hat{z}^{*}, \hat{m} ; \hat{\mu}\right) e^{-V \tilde{\mathcal{L}}_{\text {stat }}^{\pi}} \sim Z^{N_{f}=1, n=1}\left(\hat{m}, \hat{z}, \hat{z}^{*} ; \hat{\mu}\right) .
$$




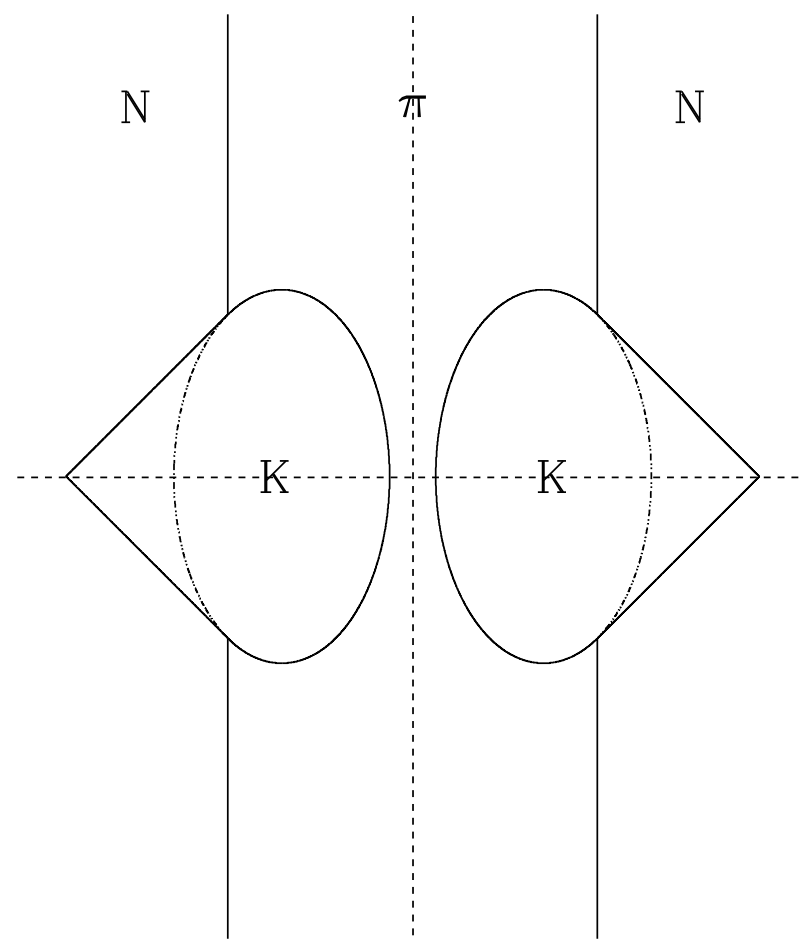

FIG. 2: Phase diagram of the Dirac spectrum in the complex $z$ plane for one dynamical flavor. The support of the quenched spectrum is between the two vertical solid black lines. Unquenching introduces the oscillations within the ellipses which intersect the $x$-axis at $x= \pm m$ and $x= \pm\left(\frac{8}{3} F_{\pi}^{2} \mu^{2} / \Sigma-m\right)$ (dashed curve). The labels $\mathrm{N}, \pi, \mathrm{K}$ refer to the normal, pion and kaon condensed phases of the generating functional for the eigenvalue density. In the region between the triangle and the ellipse the spectral density oscillates with an amplitude that is exponentially suppressed with the volume.

We introduced $\tilde{\mathcal{L}}_{\text {stat }}^{\pi}$ as formal expression for $\mathcal{L}_{\text {stat }}^{\pi}$ without taking into account the constraint $|\cos \alpha| \leq 1$. Since $Z^{N_{f}=1, n=1}\left(\hat{m}, \hat{z}, \hat{z}^{*} ; \hat{\mu}\right) \sim \exp \left[-V \mathcal{L}_{\text {stat }}^{\pi}\right]$ in the pion condensed phase, we observe that the eigenvalue density in this region is of order 1. Alternatively, this relation is necessary to obtain an eigenvalue density that does not depend exponentially on the volume the in pion condensed phase.

The mean field result for the eigenvalue density (per unit volume) is thus given by

$$
\rho_{\pi}^{N_{f}=1}\left(z, z^{*}, m ; \mu\right)=-\frac{1}{\pi} \partial_{z} \partial_{z^{*}} \mathcal{L}_{\text {stat }}^{\pi}=\frac{\Sigma^{2}}{4 \mu^{2} F^{2}} .
$$

For $\beta=\pi / 2$ the static free energy is minimized by

$$
\cos \alpha=\frac{\Sigma\left(m+z^{*}\right)}{4 \mu^{2} F^{2}} \quad \text { with } \quad \operatorname{Re} \Sigma\left(m+z^{*}\right) /\left(4 \mu^{2} F^{2}\right)<1 .
$$


This is the kaon condensed phase with free energy given by

$$
\mathcal{L}_{\text {stat }}^{K}=-2 \mu^{2} F^{2}\left(1-\cos ^{2} \alpha\right)-\Sigma\left(m+z^{*}\right) \cos \alpha-\Sigma z=-\Sigma z-2 \mu^{2} F^{2}+\frac{\Sigma^{2}\left(m+z^{*}\right)^{2}}{8 \mu^{2} F^{2}} .
$$

The condition in (35) arises from the requirement that the real part of the saddle point is inside the integration domain.

The phase boundaries occur where the real part of the Lagrangian for two different phases coincides. Notice that $x>0$. The boundary between the normal phase and the pion condensed phase is at

$$
\left(z+z^{*}\right) \Sigma=4 \mu^{2} F^{2}
$$

The boundary between the pion condensed phase and the kaon condensed phase is given by

$$
m \Sigma+\frac{\left(z+z^{*}\right)^{2} \Sigma^{2}}{8 \mu^{2} F^{2}}=x \Sigma+\operatorname{Re}\left[\frac{\left(z^{*}+m\right)^{2} \Sigma^{2}}{8 \mu^{2} F^{2}}\right] .
$$

With $z=x+i y$ this can be written as

$$
\frac{x^{2} \Sigma^{2}}{2 \mu^{2} F^{2}}-2 x \Sigma+\frac{y^{2} \Sigma^{2}}{8 \mu^{2} F^{2}}=-(x+m) \Sigma+\frac{(x+m)^{2} \Sigma^{2}}{8 \mu^{2} F^{2}},
$$

in agreement with [16]. The boundary between the normal phase and the kaon condensed phase is given by

$$
\frac{y^{2} \Sigma^{2}}{8 \mu^{2} F^{2}}=2 \mu^{2} F^{2}-(x+m) \Sigma+\frac{(x+m)^{2} \Sigma^{2}}{8 \mu^{2} F^{2}}=\frac{1}{8 \mu^{2} F^{2}}\left((x+m) \Sigma-4 \mu^{2} F^{2}\right)^{2} .
$$

This implies that kaon condensed phase dominates in the region

$$
\begin{array}{r}
2 \mu^{2} F^{2}<x \Sigma<4 \mu^{2} F^{2}-m \Sigma, \quad|y \Sigma|<4 \mu^{2} F^{2}-(x+m) \Sigma \\
m \Sigma<x \Sigma<2 \mu^{2} F^{2}, \quad \frac{y^{2} \Sigma^{2}}{8 \mu^{2} F^{2}}<-(x+m) \Sigma+\frac{(x+m)^{2} \Sigma^{2}}{8 \mu^{2} F^{2}}+2 x \Sigma-\frac{x^{2} \Sigma^{2}}{2 \mu^{2} F^{2}},
\end{array}
$$

and in a similar region that is given by the mirror image of this region with respect to the $y$-axis. Because of the relation (33) the spectral density in the kaon condensed phase is given by

$$
\rho^{N_{f}=1}\left(\hat{z}, \hat{z}^{*}, \hat{m} ; \hat{\mu}\right) \sim e^{-V \mathcal{L}_{\text {stat }}^{K}+V \tilde{\mathcal{L}}_{\text {stat }}^{\pi}}
$$

This takes into account the requirement that $|\cos \alpha| \leq 1$ in the condensed phase. The expression $\operatorname{Re}\left[\mathcal{L}_{\text {stat }}^{K}\right]<\operatorname{Re}\left[\tilde{\mathcal{L}}_{\text {stat }}^{\pi}\right]$ is a curve that splits the kaon condensed region into two parts. Inside the ellipse, the eigenvalue density grows exponentially with the volume. Since the free energy 
in the kaon condensed phase is complex, cf. (36), the eigenvalue density oscillates with a period proportional to the inverse volume. Outside of the ellipse defined by the second equation in (41) the oscillations have an exponentially small amplitude with increasing volume.

Below we will argue that the boundaries between the different phases of the Dirac spectrum do not depend on the number of replicas. However, for $n=1$ the mean field result for the free energy given by (36) results in a vanishing eigenvalue density in the oscillatory region. This implies that the resolvent for $n=1$ is discontinuous across the phase boundary between the pion condensed phase and the kaon condensed phase. We have shown numerically that the zeros of the $n=1$ partition function are located on the same ellipse.

The phase diagram in the $x y$-plane is shown in Fig. 2, The kaon condensed region is split into two parts by the dotted curve which intersects the $x$-axis at $x \Sigma=\frac{8}{3} \mu^{2} F^{2}-\frac{1}{3} m \Sigma$. Only the region that is inside the ellipse shows exponentially large oscillations. The three phases intersect each other at

$$
x \Sigma=2 \mu^{2} F^{2}, \quad|y| \Sigma=2 \mu^{2} F^{2}-m \Sigma .
$$

For $y=0$ we can distinguish the following phases [32]:

- a normal phase for $2 \mu^{2} F^{2}<x \Sigma<m \Sigma$ and $2 m \Sigma<4 \mu^{2} F^{2}<(x+m) \Sigma$.

- a pion condensed phase for $x \Sigma<2 \mu^{2} F^{2}<m \Sigma$ and $x \Sigma<m \Sigma$ with $x \Sigma>2 \mu^{2} F^{2}$.

- a kaon condensed phase for $4 \mu^{2} F^{2}>(x+m) \Sigma>2 m \Sigma$.

As we have seen in Fig. 2, the kaon condensed phase is split into two parts by the the curve

$$
x \Sigma=\frac{8}{3} \mu^{2} F^{2}-\frac{m \Sigma}{3},
$$

with exponentially large (small) oscillations in the region to the right (left) of this curve. Figure 3 shows a phase diagram of the Dirac spectrum in the $\mu^{2}-x$ plane for $y=0$ and $\hat{m}=5$. For $\mu^{2} F^{2}<m \Sigma / 2$ kaon condensed phase is absent.

The border lines between the different phases can be easily understood physically. Pion condensation occurs when $\mu_{I}>m_{\pi}$ while kaon condensation is favored when $\mu_{S}>m_{K}$ and $m_{K}<m_{\pi}$. Using (21) and (22) the condition (37) can be rewritten as $\mu_{I}=m_{\pi}$. For $y=0$ the boundary between the normal phase and the kaon condensed phase (see Eq. (40)) can be written as 


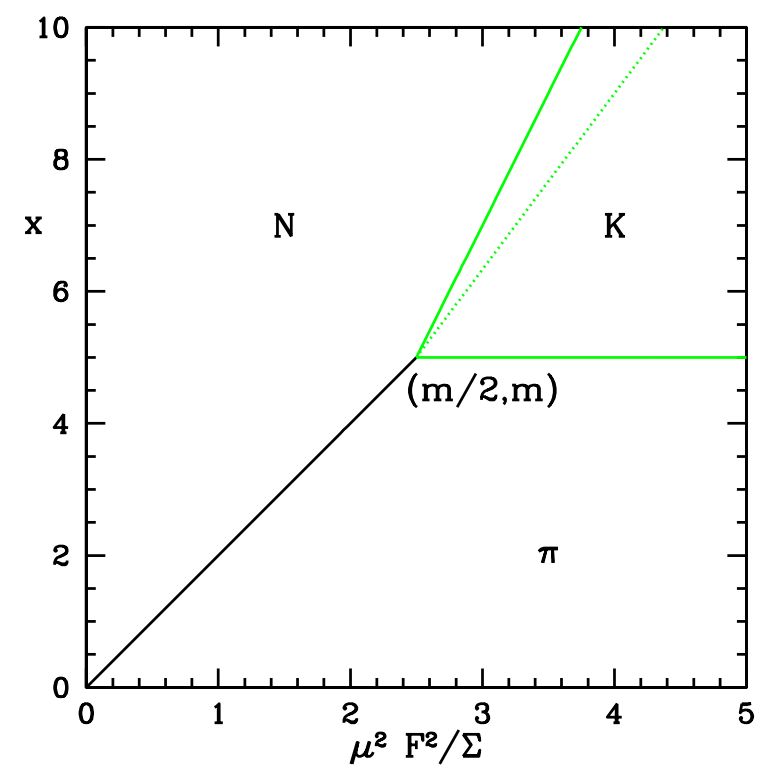

FIG. 3: Phase diagram of the Dirac spectrum in the $\mu^{2}-x$ plane. The dashed curve is the line $x=\frac{8}{3} \mu^{2} F^{2} / \Sigma$.

$\mu_{S}=m_{K} / 2$. The boundary between the pion condensed phase and the kaon condensed phase at $y=0$ (see Eq. (39) ) can be written as $m_{K}=m_{\pi}$. The reason is that since $\mu_{I}=2 \mu_{S}=\mu$, the condensed phase with the lightest boson will dominate.

\section{MEAN FIELD FREE ENERGY FOR $n$ REPLICAS}

In this section we derive the mean field free energy of the partition function (17). It is obtained from the free energy of the static chiral Lagrangian, which is still given by the static part of (23) but now $U \in U(2 n+1)$. The baryon charge matrix and the mass matrix are now given by

$$
\begin{aligned}
B_{\nu} & =\delta_{\nu, 0} \operatorname{diag}\left(\mathbf{1}_{\mathbf{n}},-\mathbf{1}_{\mathbf{n}}, 1\right), \\
\mathcal{M} & =\operatorname{diag}\left(z \mathbf{1}_{\mathbf{n}}, z^{*} \mathbf{1}_{\mathbf{n}}, m\right),
\end{aligned}
$$

where $\mathbf{1}_{\mathbf{n}}$ is the $n \times n$ unit matrix. 
The ansatz for the vacuum configurations is given by

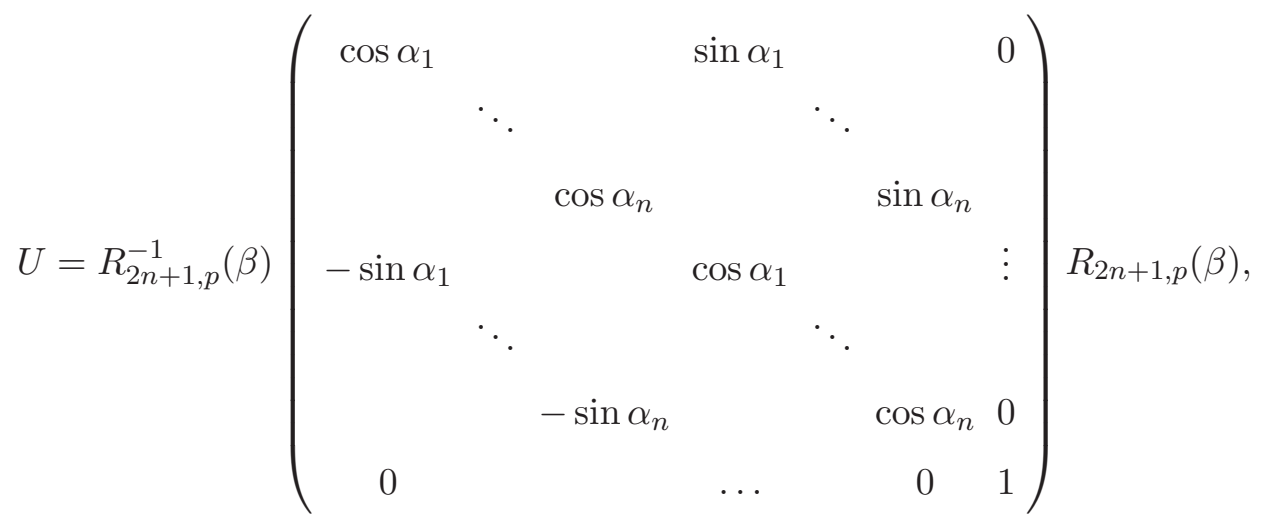

where $R_{2 n+1, p}(\beta)$ is a rotation by $\beta$ in the $2 n+1, p$-plane for some choice of $p$.

In the normal phase all $\alpha_{k}=0$ so that $U=1$ and

$$
\mathcal{L}_{\text {stat }}^{N}=-\Sigma\left(m+n z+n z^{*}\right)
$$

For $\alpha_{k} \neq 0$, the minimum of $\mathcal{L}_{\text {stat }}$ is either at $\beta=0$ or at $\beta=\pi / 2$. For $\beta=0$ we have

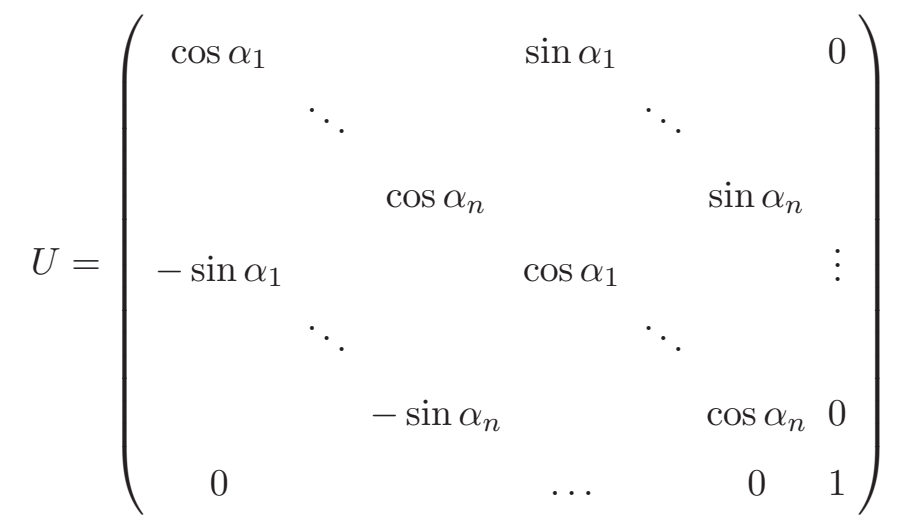

and find

$$
\mathcal{L}_{\text {stat }}^{\pi}=-m \Sigma-\sum_{k=1}^{n}\left[2 \mu^{2} F^{2}\left(1-\cos ^{2} \alpha_{k}\right)+\Sigma\left(z+z^{*}\right) \cos \alpha_{k}\right] .
$$

The saddle point equation for each of the replicas is the same with nonzero solution given by

$$
\cos \alpha_{k}=\frac{\Sigma\left(z+z^{*}\right)}{4 \mu^{2} F^{2}} .
$$

This is the pion condensed phase with free energy given by

$$
\mathcal{L}_{\text {stat }}^{\pi}=-\Sigma m-2 n \mu^{2} F^{2}-n \frac{\Sigma^{2}\left(z+z^{*}\right)^{2}}{8 \mu^{2} F^{2}} .
$$

For $\beta=\pi / 2$, the ansatz (47) for the vacuum configuration depends on $p$. To excite mesons with a nonzero isospin charge we necessarily have that $1 \leq p \leq n$. For $k \neq p$ the saddle point equation 
is again given by (51) and is the same as in the pion condensed case. For $k=p$ the saddle point equation is given by (35) found for $n=1$. For arbitrary $n$ we thus find that the free energy for the kaon condensed phase is given by

$$
\mathcal{L}_{\text {stat }}^{K}=-\Sigma z-2 n \mu^{2} F^{2}-(n-1) \frac{\Sigma^{2}\left(z^{*}+z\right)^{2}}{8 \mu^{2} F^{2}}-\frac{\Sigma^{2}\left(z^{*}+m\right)^{2}}{8 \mu^{2} F^{2}} .
$$

\section{A. Phase Boundaries}

It is immediately clear that the phase boundaries between the normal phase and the pion condensed phase and between the pion condensed phase and the kaon condensed phase do not depend on $n$. However, the boundary between the kaon condensed phase and the normal phase does depend on $n$. Also for general $n$ the kaon condensed region is split into a region with a spectral density that grows exponentially with the volume, and a region where it decreases exponentially with the volume. Because the spectral density in the pion condensed region does not depend exponentially on the volume for $n$ replicas, we also have the relation

$$
\rho_{\nu}^{N_{f}=1, n}\left(\hat{z}, \hat{z}^{*}, \hat{m} ; \hat{\mu}\right) e^{-V \tilde{\mathcal{L}}_{\text {stat }}^{\pi}} \sim Z^{N_{f}=1, n}\left(\hat{m}, \hat{z}, \hat{z}^{*} ; \hat{\mu}\right)
$$

This implies that the boundary of the oscillatory region with an exponentially large amplitude is given by the equality of the free energies of the pion condensed phase and the normal phase and therefore does not depend on $n$.

\section{CONCLUSIONS}

The Dirac spectrum for QCD with dynamical quarks at nonzero quark chemical potential can be split into three regions. A region with no eigenvalues, a region with a constant eigenvalue density equal to the quenched spectral density, and a region where the spectral density shows oscillations. This last region is further split into one region with an exponentially large and one region with an exponentially suppressed amplitude. These regions have been derived from the chiral Lagrangian for the generating functional of the eigenvalue density. The generating functional includes a conjugate quark and this makes it possible to reinterpret the chemical potential as a nonzero isospin and strangeness chemical potential. The three regions of the eigenvalue density correspond to a normal phase, a pion condensed phase and a kaon condensed phase of the generating functional, respectively. 
These results were derived from the leading order chiral Lagrangian in the mean field limit which is valid for $m,|z| \ll F^{4} / \Sigma$ and $\mu \ll F$. However, we expect that the Dirac spectrum beyond this domain will behave in a similar fashion.

The mean field limit is the lowest order term in the standard expansion of chiral perturbation theory [33]. The results presented here thus form the basis for an analysis that contains loop effects as well as higher order terms in the chiral Lagrangian. The inclusion of loop effects in chiral perturbation theory at nonzero chemical potential is well defined [34] and can be extended to include the effects of a nonzero temperature as well [35, 36].

Acknowledgments: We wish to thank Poul Henrik Damgaard for useful discussions. This work was supported in part by U.S. DOE Grant No. DE-FG-88ER40388.

[1] T. Banks and A. Casher, Nucl. Phys. B169, 103 (1980).

[2] I. Barbour, N. E. Behilil, E. Dagotto, F. Karsch, A. Moreo, M. Stone and H. W. Wyld, Nucl. Phys. B 275, 296 (1986).

[3] P. E. Gibbs, Glasgow preprint 86-0389 (unpublished).

[4] C. Baillie, K. C. Bowler, P. E. Gibbs, I. M. Barbour and M. Rafique, Phys. Lett. B 197, 195 (1987).

[5] J. C. Vink, Nucl. Phys. B 323, 399 (1989).

[6] C. T. H. Davies and E. G. Klepfish, Phys. Lett. B 256, 68 (1991).

[7] M. P. Lombardo, J. B. Kogut and D. K. Sinclair, Phys. Rev. D 54, 2303 (1996) arXiv:hep-lat/9511026.

[8] T. Schafer, Phys. Rev. D 57, 3950 (1998) arXiv:hep-ph/9708256.

[9] H. Markum, R. Pullirsch and T. Wettig, Phys. Rev. Lett. 83, 484 (1999) arXiv:hep-lat/9906020.

[10] E. Bittner, S. Hands, H. Markum and R. Pullirsch, Prog. Theor. Phys. Suppl. 153, 295 (2004) arXiv:hep-lat/0402015.

[11] D. Toublan and J. J. M. Verbaarschot, Int. J. Mod. Phys. B 15, 1404 (2001) hep-th/0001110.

[12] T. D. Cohen, Phys. Rev. Lett. 91, 222001 (2003) arXiv:hep-ph/0307089.

[13] M. A. Stephanov, Phys. Rev. Lett. 76, 4472 (1996) arXiv:hep-lat/9604003.

[14] J. C. Osborn, Phys. Rev. Lett. 93, 222001 (2004), arXiv:hep-th/0403131.

[15] G. Akemann, J. C. Osborn, K. Splittorff and J. J. M. Verbaarschot, Nucl. Phys. B 712, 287 (2005), arXiv:hep-th/0411030.

[16] J. C. Osborn, K. Splittorff and J. J. M. Verbaarschot, Phys. Rev. Lett. 94, 202001 (2005), arXiv:hep-th/0501210.

[17] J. C. Osborn, K. Splittorff and J. J. M. Verbaarschot, arXiv:0805.1303 [hep-th].

[18] E. V. Shuryak and J. J. M. Verbaarschot, Nucl. Phys. A 560, 306 (1993) arXiv:hep-th/9212088.

[19] J. J. M. Verbaarschot, Phys. Rev. Lett. 72, 2531 (1994) arXiv:hep-th/9401059. 
[20] J. J. M. Verbaarschot, Phys. Lett. B 368, 137 (1996) arXiv:hep-ph/9509369.

[21] J. Gasser and H. Leutwyler, Phys. Lett. B 184, 83 (1987).

[22] K. Splittorff and J. J. M. Verbaarschot, Nucl. Phys. B 683, 467 (2004); arXiv:hep-th/0310271].

[23] G. Akemann, J. Bloch, L. Shifrin and T. Wettig, Phys. Rev. Lett. 100, 032002 (2008) arXiv:0710.2865 [hep-lat]]; G. Akemann and P. H. Damgaard, JHEP 0803, 073 (2008) arXiv:0803.1171 [hep-th]].

[24] G. Akemann and T. Wettig, Phys. Rev. Lett. 92, 102002 (2004) [Erratum-ibid. 96, 029902 (2006)] arXiv:hep-lat/0308003.

[25] J. C. Osborn and T. Wettig, PoS LAT2005, 200 (2006) arXiv:hep-lat/0510115.

[26] J. Bloch and T. Wettig, PoS LAT2006, 206 (2006) arXiv:hep-lat/0609020.

[27] J. C. Osborn, K. Splittorff and J. J. M. Verbaarschot, Int. J. Mod. Phys. A 21, 859 (2006) arXiv:hep-ph/0509263.

[28] V.L. Girko, Theory of Random Determinants, Springer, 1990.

[29] E. Kanzieper, Phys. Rev. Lett. 89, 250201 (2002) arXiv:cond-mat/0207745.

[30] K. Splittorff and J. J. M. Verbaarschot, Phys. Rev. Lett. 90, 041601 (2003) arXiv:cond-mat/0209594.

[31] D. T. Son and M. A. Stephanov, Phys. Rev. Lett. 86, 592 (2001) arXiv:hep-ph/0005225.

[32] D. Toublan and J. B. Kogut, Phys. Lett. B 605, 129 (2005) arXiv:hep-ph/0409310.

[33] J. Gasser and H. Leutwyler, Annals Phys. 158, 142 (1984).

[34] K. Splittorff, D. Toublan and J. J. M. Verbaarschot, Nucl. Phys. B 620, 290 (2002) arXiv:hep-ph/0108040.

[35] K. Splittorff, D. Toublan and J. J. M. Verbaarschot, Nucl. Phys. B 639, 524 (2002) arXiv:hep-ph/0204076.

[36] G. V. Dunne and S. M. Nishigaki, Nucl. Phys. B 670, 307 (2003) arXiv:hep-ph/0306220. 